\title{
ENSEÑAR HISTORIA, UN RETO ENTRE LA DIDÁCTICA Y LA DISCIPLINA: REFLEXIÓN DESDE LA FORMACIÓN DE DOCENTES DE CIENCIAS SOCIALES EN COLOMBIA
}

\author{
TEACH HISTORY, A CHALLENGE BETWEEN THE DIDACTIC AND THE \\ DISCIPLINE: REFLECTION SINCE THE FORMATION A SOCIAL SCIENCE \\ TEACHERS IN COLOMBIA
}

\author{
Paula Tatiana Pantoja Suárez*
}

\begin{abstract}
La intención del presente artículo es establecer una reflexión desde los aspectos disciplinar y didáctico en la enseñanza de la Historia, que surgieron como parte de una investigación con docentes de Ciencias Sociales en formación de la Universidad de Caldas (Manizales, Colombia), alrededor de la categoría conciencia histórica. La investigación fue realizada desde el enfoque de la teoría fundamentada, que desde discursos y prácticas de docentes en ejercicio y formación de la mencionada institución, permitieron construir reflexiones acerca del rol social de la historia y de la historia en la escuela. Este trabajo expresa la necesidad que tienen los programas de formación de maestros para relacionar en sus procesos de enseñanza los saberes propios que soportan los campos del conocimiento, con las intencionalidades formativas que soportan los procesos que se realizarán en su vida como profesionales de la enseñanza de las Ciencias Sociales bajo el contexto colombiano.
\end{abstract}

Palabras claves: Licenciados en Ciencias Sociales, enseñanza de la Historia, saber disciplinar, saber didáctico.

The intention of this article is to establish a reflection from the disciplinary and didactic aspects on the teaching of History, which emerged as part of an investigation with teachers of Social Sciences in formation of the University of Caldas (Manizales, Colombia), around the historical consciousness category. The research was carried out from the approach of the theory grounded, where from the speeches and practices of teachers in the exercise and formation of the mentioned institution, allowed make reflections on the social role of history and history in school. This work expresses the need for teacher training programs to relate in their teaching processes their own knowledge that supports the fields of knowledge, with the formative intentions that support the processes that will be realized in their life as teaching professionals of the Social Sciences under the Colombian context.

Key words: Teachers in Social Sciences, Teaching History, disciplinary knowledge, didactic knowledge.

Creo que hay un futuro para la enseñanza del pasado, aunque su razón de ser sería un poco diferente de las tradicionales. No como un juez. universal del bien y del mal (...) Más bien, es un manera de ofrecer una cuenta para entender el presente, un camino privilegiado para la comprensión de la sociedad, y lo que es quizás más importante una manera de abrir los ojos a los valores y formas de vida y la manera de sensibilizar nuestras realidades del cambio y nos proporciona las herramientas para explicarlo, tratar con él y aceptarlo. Michael Heyd (2012)

\section{Introducción}

La Historia responde, entre muchos intereses, a una explicación de los sucesos de otros tiempos desde el presente y a su vez es la búsqueda de los humanos por comprender su hoy desde el acontecer pasado. Por tanto, según plantea Zemelman (2007: 37), "[... ] la movilidad de las sociedades pasadas y presentes otorgan a los fenómenos históricos y a los procesos de enseñanza del conocimiento histórico una posibilidad diversa de interpretación, análisis y contraste, pues estudiar historia es intentar interpretar en la multiplicidad humana un punto de encuentro y reflexión de las acciones pasadas y aquello que tratan de comprender desde la actualidad sobre las mismas [...]". Esta situación ha planteado que la objetividad "pura" de la Historia no puede delimitarse desde la separación del sujeto que estudia con el objeto estudiado, pues ambos son sujetos culturales, espaciales y temporales y por tanto existen puntos de encuentro que ligan al investigador con su propia esencia y generan interpretaciones.

En tal sentido, según Meyer, E. (2000: 84), “[...] se persiguen nuevas alternativas frente a la historia

* Universidad de Caldas, Manizales-Colombia. Correo electrónico: paula.pantoja@ucaldas.edu.co 
tradicional, acercando los límites de la historia a los de las vidas de las personas, con el fin de que la historia se humanice y, por ende, resulte más próxima y comprensible. Se trata de extender el sustento de la historia, ampliar su ámbito de estudio, aprovechar nuevas materias primas y ofrecer nuevos mapas de conocimiento [...]".

Ideas como las anteriores se relacionan con una postura denominada como Historia Social, la que reconoce en la ciencia histórica una rigurosidad en términos metodológicos, pero que no puede dedicarse exclusivamente a la descripción de hechos, pues como cualquier campo del saber humano, tiene un compromiso con la sociedad plural del pasado y el presente, situación que el investigador no puede negar y que otorga a la Historia una búsqueda por las interpretaciones, no por verdades absolutas. La premisa anterior es comprendida por la gran mayoría de historiadores y estudiantes universitarios, sin embargo en las aulas de clase de la educación básica y media, pareciese que la Historia es una verdad estática, única e irrefutable. ${ }^{1}$ Aunque el caso no se puede generalizar, sí es una situación que debe propiciar reflexiones y cambios en la labor de formación del docente de Ciencias Sociales, quien debe buscar en la Historia ese punto de encuentro planeado y el sentido del saber en los problemas socialmente vivos, para "dotar de sentido a los acontecimientos históricos" (Santisteban, A., 2010: 46).

Estos elementos fueron el fundamento para iniciar una investigación que tiene como premisa fundamental que saber y enseñar Historia son dos procesos complementarios pero diferentes, por ello es importante realizar ciertas diferenciaciones que le permitan al docente de Ciencias Sociales y de Historia una conceptualización desde las realidades propias de la Universidad de Caldas, en contraste con procesos de análisis de posturas y perspectivas que se han desarrollado respecto del porqué y el cómo conocer la historia humana. La reflexión mencionada es un pilar real en la dicotomía entre saber Historia y saber enseñarla, porque el verdadero discípulo de Clío debe poder establecer el acto comunicativo concerniente a los legados de diferentes momentos y culturas y, al igual que las demás musas, debe inspirar con sus palabras y acciones en la re-creación crítica de los tiempos humanos. Esta comprensión de las culturas tiene un sentido en la medida que se establecen conexiones con el territorio vivo y las dinámicas de comprensión temporal a partir las fronteras cambiantes y la manera de dar sentido a los espacios.

La enseñanza de la Historia requiere enfrentarse a la complejidad del conocimiento histórico, utilizando modelos explicativos, comprensivos y propositivos para la caracterización de la Historia escolar. En el contexto de la Universidad de Caldas, los estudiantes y docentes relacionan el debate entre saber Historia y saber enseñarla como una reflexión que está atravesada por el tema de la formación disciplinar y profesional. Estas reflexiones presentan puntos de encuentro y desencuentro con los referentes nacionales para la enseñanza de las Ciencias Sociales en Colombia, a partir de la propuesta de formación por competencias, donde aparecen los estándares y lineamientos que se deben alcanzar por medio de esta área escolar, que trabaja de manera integrada la Historia, la Geografía, las Ciencias Políticas y la Economía.

\section{Metodología}

En el trayecto del proceso investigativo, la metodología seleccionada para el desarrollo de la investigación fue la teoría fundamentada, entorno que explica una integración entre el trabajo teórico y práctico como eje para proponer una teoría sustantiva basada en el interés y el problema objeto de estudio. La intención de aportar a los procesos de formación docente en ciencias sociales es uno de los objetivos centrales de la investigación a partir de dos ejes transversales: la conciencia histórica y la historia regional, como una posibilidad para aportar a la formación y construcción de comunidades académicas que discutan acerca de la complejidad y los retos de la formación de docentes en ciencias sociales y particularmente en la enseñanza de estas.

El análisis documental como estrategia de análisis desde la postura metodológica trabajada, se desarrolló basado los aportes de Sandoval (1996). Para el desarrollo de los antecedentes, el estado del arte y los referentes teóricos de las categorías de trabajo también fueron seleccionados en esta etapa del análisis documental no con el fin de crear supuestos previos al análisis de la información, sino con el interés de contextualizar la investigación.

Como parte de este proceso, las entrevistas en profundidad son uno de los instrumentos más empleados en el enfoque de la teoría fundamentada, ya que permiten mayor nivel de ampliación en los discursos de las personas respecto de situaciones 
o problemas desde un contexto específico, estas fueron aplicadas a docentes del departamento de Historia y Geografía de la Universidad de Caldas con experiencia en procesos de enseñanza e investigación en historia regional, además de estudiantes de la Licenciatura en Ciencias Sociales en diferentes momentos de su proceso de formación universitaria. Fragmentos de estas entrevistas se encuentran en el contenido del artículo, no como un elemento unificador de discursos, sino como una posibilidad de exaltar las reflexiones de personas que viven la enseñanza de la historia desde diferentes roles, sea como formador de formadores o como un estudiante que piensa en su futura práctica profesional.

\section{Contexto actual acerca de los estudios para la enseñanza de los hombres en el tiempo}

Uno de los pilares de la Historia actual es la preocupación por reflejar en su enseñanza un proceso de mayor envergadura que la neta memorización de hechos y fenómenos realizados por algunos personajes específicos en fechas exactas ${ }^{2}$. En atención a ello, han surgido diferentes posturas que piensan en otras alternativas para aproximarse a la Historia y su enseñanza, ya sea en relación con las transformaciones de los sistemas educativos o perspectivas de la ciencia histórica en situaciones sociales, políticas y culturales ${ }^{3}$. Por tanto, conocer los avances teóricos e investigativos en torno a las múltiples posturas de la enseñanza de la Historia es relevante, ya que no permite establecer claridades en torno a la posibilidad de tener una mejor comprensión y apropiación de las temáticas propias para esta área del conocimiento. La intención de contextualizar un estado del arte no es crear supuestos, sino aportar al debate académico y práctico en la enseñanza de la Historia y las Ciencias Sociales. ${ }^{4}$

En el campo internacional se desarrollan diferentes posturas que es importante conocer para crear puntos de encuentro, no bajo la pretensión de replicar modelos extranjeros, sino de la construcción de comunidad académica. La propuesta de la Didáctica de la Historia Alemana ${ }^{5}$ se ha dedicado desde la década de los noventa al estudio de la enseñanza de la Historia como parte de la ciencia histórica, la que concibe su enseñanza como una función orientadora vital que busca desmitificar y deconstruir realidades (Valls, R., 1999), cuyos autores más destacados son Jörn Rüssen y Jürgen Kocka. Estos referentes han servido como base para estudios de la enseñanza de la Historia no solo en Alemania, sino en el resto de Europa (2002) y Canadá, donde el mismo Rüssen propuso el centro para la Conciencia Histórica ${ }^{6}$ en los procesos de formación de docentes de Historia y Estudios Sociales ${ }^{7}$.

En el mismo contexto europeo se destacan los trabajos de Pagés, J. y Santisteban, A. y González, N. (2010), quienes han desarrollado varias propuestas para la enseñanza de la Historia, en el marco de la Maestría y el Doctorado en Didáctica de las Ciencias Sociales. Ellos definen el aprendizaje del tiempo como un proceso complejo, que solo se entiende a partir de un análisis amplio y transdisciplinario, con el fin de hallar en la Historia ciertos significados para comprender mejor el presente de un pasado y aprender a intervenir en el futuro de un presente.

Pagés, J. (2004) reflexiona acerca de la vida y las acciones humanas a lo largo de un desarrollo temporal, en un tiempo que se vive y se experimenta personalmente pero que está inmerso en un tiempo social que lo enmarca, lo contextualiza y lo explica. Estos referentes deben ser comprendidos por el futuro docente de Historia, quien tiene en sus manos la responsabilidad no de educar historiadores, sino de contribuir a la formación para el ejercicio de la ciudadanía.

De esta experiencia emana una cierta conciencia de temporalidad, de percepción mental del tiempo, que se modifica con la edad y nuevas experiencias. $\mathrm{La}$ temporalidad constituye un elemento fundamental de la personalidad individual y es un aspecto esencial, por esta razón debería formar parte de los objetivos de la enseñanza y más concretamente de la enseñanza de las Ciencias Sociales y en particular de la Historia. Esta postura tiene como eje una relación con elementos de formación de ciudadanía, en aras de promover interés en los estudiantes para conocer la Historia, por medio de una enseñanza que no se quede solo en el pasado o una simple sucesión de hechos, sino como un proceso complejo y dinámico donde los diferentes planos de la realidad social se interrelacionan, explicándose mutuamente en un aquí, un antes y un después.

El español Carretero, M. (2006) se ha dedicado al estudio de la enseñanza de la Historia desde la Didáctica y la memoria colectiva, con la intención de generar profundas transformaciones y debates. $\mathrm{La}$ escuela es justamente un ámbito donde las sociedades se disputan las memorias posibles sobre sí mismas. 
Carretero y Kriger (2010: 58) concluyen que, "el interés por la función política de la historia escolar parece haber crecido desproporcionadamente en relación con el interés por su función pedagógica, propiciando algunos usos incorrectos del pasado". Plantea además la importancia de "...distinguir entre el pensamiento historiográfico de los especialistas y su eventual uso en la esfera educativa asignándoles a los historiadores el rol de custodios del pasado y fundamentalmente de la conciencia histórica en el presente". De igual manera reconoce que “... son cruciales las implicaciones y deslizamientos de esta discusión historiográfica al campo de la enseñanza escolar, donde plantear el problema de los usos correctos o incorrectos de la historia implica poner el foco precisamente en las articulaciones entre la gestión del pasado y la construcción de un presente común". Los aspectos anteriores invitan entonces a repensar la forma como se orienta la historia en las instituciones, para dejar de ser vista solo como la posibilidad de aportar a la formación de ciudadanos y se justifique más desde el rol que tienen la enseñanza de la historia en la construcción de identidad, conciencia y pensamiento.

En el contexto español, Prats, J. (2001: 16) define los principales objetivos de enseñanza de la Historia: "Comprender los hechos ocurridos en el pasado y saber situarlos en su contexto, comprender que en el análisis del pasado hay muchos puntos de vista diferentes, comprender que hay formas muy diversas de adquirir, obtener y evaluar informaciones sobre el pasado y ser capaces de transmitir de forma organizada lo que sobre el pasado se ha estudiado o se ha obtenido". Dichos objetivos, expone Prats (2001: 15), tienen el propósito fundamental de "Facilitar la comprensión del presente, Preparar a los alumnos para la vida adulta, Despertar el interés por el pasado, Potenciar en los niños y adolescentes un sentido de identidad, Ayudar a los alumnos en la comprensión de sus propias raíces culturales y de la herencia común, Contribuir al conocimiento y comprensión de otros países y culturas del mundo de hoy, Contribuir a desarrollar las facultades de la mente mediante un estudio disciplinado, Introducir a los alumnos en el conocimiento y dominio de una metodología rigurosa propia de los Historiadores y Enriquecer otras áreas del currículum".

En el marco de América Latina, los argentinos han desarrollado varias posturas en la comprensión de la Historia; ${ }^{8}$ el estudio de Nosei, M. (2007: 75) plantea que en el proceso de enseñanza y comprensión de la historia existe un estado de riesgo pedagógico, entendido este como aquel que señala limitaciones respecto de una construcción significativa del saber. De otra parte, las profesoras Camillioni, A. y Aisenberg, B. (1996) han desarrollado elementos de la Didáctica de las Ciencias Sociales y la Didáctica de la Historia con la intencionalidad principal de construir posturas políticas en defensa de los derechos humanos y las responsabilidades desde y para el Estado. Asimismo, el historiador argentino Rivero, R (2004) sostiene que la “...función de la enseñanza de la Historia tiene que ver, por un lado, con la necesidad de favorecer una conciencia crítica ciudadana y, por el otro, con el objetivo identitario de forjar y mejorar los vínculos representacionales entre los miembros de determinada comunidad social y nacional" (Rivero, R., 2004:47).

De otra parte, Camilloni, A. (1994) promueve para el área de las Ciencias Sociales y específicamente para la Historia, una enseñanza significativa y una propuesta de trabajo basada en las lógicas de la metodología de la investigación, en la que se da a conocer la experiencia a partir del análisis de lo que sucede en el contexto educativo; específicamente en los niveles educativos obligatorios, en los que la enseñanza de la Historia conserva una concepción positivista expresada en la anacronía, linealidad y tratamiento descriptivo de los hechos históricos. Esta situación evidencia el desfase de los contenidos que se enseñan con los desarrollos de las disciplinas a las que corresponden, como una crisis que requiere una reflexión y acercamiento teórico-metodológico en la Didáctica de las Ciencias Sociales.

Para el caso mexicano, Sebastián Plá (2005) destaca el aula como un espacio de relaciones, con una intencionalidad formativa, donde convergen varios tipos de saberes del docente, de los estudiantes y el fruto de esta interacción, pero considerando de particular importancia la articulación entre investigación y enseñanza de la historia,${ }^{9}$ si se quiere enseñar a pensar históricamente. En este mismo contexto Lima, Bonilla y Arista (2010) reconocen como una de las dimensiones de la enseñanza de la Historia en la educación básica el aprender a pensar históricamente, lo que significa que hay que preguntarse el porqué de la situación actual y cómo se sitúan los sujetos en la sociedad. Para ello es necesario superar el presentismo, una postura que delimita la perspectiva individual y social a un corto plazo. 
En Costa Rica es representativo el trabajo de Mangano, F. (2009) respecto de las contradicciones y discontinuidades en la enseñanza de la Historia en la construcción del sujeto social, que implica problemas y discusiones en cuanto al uso y pertenencia de categorías y conceptos. También los estudios de Solano, E. (2006) presentan la Didáctica de la Historia como una ciencia social aplicada, con el fin de emplearse en los diversos niveles de enseñanza, alejados de la repetición y memorización de datos.

En el contexto colombiano existe un importante rastreo temporal de los procesos de enseñanza de la Historia. Para Guerrero, C. (2011) esta se difuminó en el escenario escolar representada ahora por la enseñanza de las Ciencias Sociales, bajo un proceso que continuó alimentando brechas e inconsistencias entre los debates epistemológicos, los modelos pedagógicos, las políticas educativas y las cotidianidades en el aula de clase, donde la enseñanza buscaba integrar las disciplinas sociales, con activismos y con multiplicidad de formas que carecían de profundidad, análisis, crítica y dominio de los temas significativos para la formación integral de los estudiantes.

La enseñanza de la historia en Colombia, según Guerrero, C. (2011:4), "ha atravesado por procesos complejos en la formación de nación -con grandes dificultades en su organización económica, política y social- que han alterado los ejercicios y hábitos de memoria personal y colectiva $\mathrm{y}$, por tanto, han repercutido en la formación de identidad. El olvido, la amnesia temporal o definitiva y la marginalización de la historia como disciplina -y de su práctica pedagógica- han constituido la herramienta para afrontar las grandes dificultades que han azotado a la sociedad colombiana". En tal sentido, es evidente que los procesos de enseñanza de la historia en Colombia han propendido por el rescate de la identidad y el reconocimiento de las diversas dificultades que el país ha pasado desde el reconocimiento de su independencia.

En tal sentido, un aspecto que ha cobrado importancia en el contexto de la enseñanza de la historia en Colombia es la responsabilidad de incluir en los proceso de aula, en los diferentes niveles del sistema educativo, la memoria y los relatos de los grupo étnicos, en atención a la diversidad cultural con la que cuenta el país, seguramente con la intención de rescatar la identidad nacional, y rememorar la forma cómo se fueron constituyendo las comunidades que hoy se asientan en el territorio nacional. Para cumplir dicho propósito, dice la historiadora Mena, M. (2009), las nuevas narraciones que conforman la historia nacional deben incluir a la multiplicidad de voces, actores y escenarios, que develen los recuerdos que en la institución educativa se vuelven difíciles de promover e incluso de activar, como los hechos de la conquista de los pueblos originarios de América.

Asimismo, es necesario resaltar que en Colombia la enseñanza de la historia en los planteles educativos colombianos se integró al área de las ciencias sociales a partir de 1984, con la expedición del Decreto 1002, con la intención de brindar una mirada integral y por demás inter y transdisciplinar, ${ }^{10}$ que permitiera, según se expone en el decreto en mención, una mejor comprensión. ${ }^{11}$ En la tesis de Guerrero, C. (2011: 166) expone que esta situación generó “... que académicos, docentes e incluso el gobierno a través del MEN permaneciera en silencio aún más agudo sobre la enseñanza de las ciencias sociales y el papel de la historia ante dicha integración. Dicha omisión obedecía a la dificultad que resultó - para los diferentes actores del escenario educativo- entender la integración, pues para aquel entonces no se contaba con las herramientas para abordarla, criticarla, explicarla o implementarla. Por tal motivo, no fue posible contar con una propuesta clara, coherente, argumentada y sólida en aspectos epistemológico, pedagógico y cognitivo ni para la enseñanza de la historia, ni para el área de ciencias sociales". Lo anterior da cuenta de la necesidad urgente de contar con académicos e investigadores que se preocupen por consolidar el campo de la enseñanza de la historia y rescaten el alto valor formativo que tiene en los procesos educativos de los escolares en los diferentes niveles.

Desde la Universidad Pedagógica Nacional, el trabajo de Rodríguez (2012) aborda las relaciones entre la memoria y la enseñanza de la historia reciente en un ámbito de formación de profesores en torno a trabajos acerca del conflicto armado colombiano. Para la autora, en dichos ejercicios investigativos se evidencia la existencia de memorias dominantes que impiden la legitimización en el escenario público de las memorias de grupos que han sido silenciados.

Trabajos como el realizado por Arias (2014) en la Universidad Distrital realizan un rastreo histórico de la enseñanza de la Historia en Colombia, con el fin de mostrar este cuerpo de conocimientos como un apetecido campo susceptible de ser capitalizado por 
distintas lógicas de saber y de poder. Esto con el fin de mostrar, que para el caso de las ciencias sociales escolares, la forma en que se divulgan los discursos políticos, académicos y periodísticos, contribuyen a crear representaciones de verdad y no a ponderar los discursos para crear escalas de los mismos.

Tal vez uno de los esfuerzos más importantes se dan en la Red de Grupos de Investigación en Didáctica de las Ciencias Sociales ${ }^{12}$ que involucra a las Universidades de Antioquia, Tecnológica de Pereira, Valle y Universidad de Caldas, en la que se han desarrollado trabajos significativos de la enseñanza de la Historia, entre estas se destaca el trabajo realizado por Villa (2013) acerca del desarrollo de la crítica histórica como parte de la formación en Ciencias Sociales. Dentro de este grupo es necesario resaltar desde lo local el trabajo realizado por Posada (2009), quien bajo un estudio de caso realiza un análisis de la enseñanza de la Historia en la Licenciatura en Ciencias Sociales de la Universidad de Caldas, resultados entre los que se destaca:

"una resignificación en donde se conceptualice a la sociedad dentro de un proceso histórico en el que cada situación se manifiesta como fruto de un antagonismo dialéctico. Por lo tanto, en la construcción del conocimiento del proceso social, el mismo análisis que se efectúa de ese proceso debe ser comprendido, y entendido, como una posible crítica de sí mismo. Donde el estudiante no puede detenerse ante la realidad, ni limitarse a ella, puede analizarla, proponer la crítica y la solución como complementos inseparables" (p. 172).

A nivel local es interesante el trabajo desarrollado en el marco de la Maestría en educación de la Universidad de Caldas desarrollado por Márquez, M. (2009: 210 ); en donde plantea que la enseñanza de la historia en Colombia juega un papel trascendental, en virtud de la situación que vive en la actualidad y, por tanto, es posible a partir de esta área contribuir desde de la enseñanza de la historia no solo en el reconocimiento del conflicto armado en el país, sino también pensar en opciones de solución del mismo en Colombia, si se estudian las maneras adecuadas para comprender cómo debe ser la enseñanza de la historia del conflicto armado y lograr así cooperar en la indagación de salidas a las problemas que trae consigo la confrontación armada. En tal perspectiva, postula la autora que el reconocimiento del estado del arte de la enseñanza de la historia, parte de reconocer que, "[...] uno de los aspectos más relevantes en la enseñanza de la historia es lograr narrar pero además interpretar los hechos y entender las realidades sociales, políticas y económicas como forma eficaz de multiplicar el conocimiento y cumplir con la función básica del maestro de enseñar la verdad y acercar al alumno de forma clara y objetiva a esa verdad" (Márquez, M. 2009: 214).

El panorama actual de las investigaciones respecto de la enseñanza de la Historia, con sus diferentes vertientes y enfoques, muestra un amplio interés por construir saber didáctico desde las intencionalidades formativas de este campo del saber humano, que tiene no solo un rol "civilizador", sino que presenta retos y roles sociales dentro de la formación de ciudadanos en la escuela. Algunas de estas reflexiones hacen parte de los procesos de los docentes de Ciencias Sociales en ejercicio y formación dentro de la Universidad de Caldas y por esta razón es vital tenerlas en cuenta, pues aunque los elementos en cada contexto podrán variar, muchos de los intereses son comunes a diferentes espacios y lugares de formación.

\section{Historia "sabia" e Historia enseñada: discursos no siempre convergentes ${ }^{13}$}

El recorrido por las posturas en torno a la Historia, con los debates que implican en las definiciones por la verdad histórica, los métodos y fuentes del quehacer historiográfico o las posturas que debe asumir el investigador en torno al saber y su relación con la sociedad, presentan una implicación directa con las conceptualizaciones realizadas de la enseñanza de la Historia, vista no únicamente como los procesos de formación de historiadores, sino desde su enseñanza en los niveles básicos de educación. ¿Es esto verdad? ¿Las trasformaciones en la manera de percibir y narrar el tiempo humano siempre repercuten en las formas de enseñarla?

La respuesta a estas cuestiones presenta el mismo carácter divergente de las escuelas de pensamiento, principalmente entre las luchas de posturas ligadas al positivismo y las relacionadas con la Historia de los Annales. Las universidades y profesores de Historia varían entre sí por las respuestas y en este 
debate se encuentra una de las principales riquezas del estudio acerca de las narrativas históricas y la temporalidad. Allí se encuentra implícita la cuestión ¿es lo mismo formar un historiador que un profesor de Historia? Teniendo en cuenta que uno de los principales campos de acción del licenciado en Ciencias Sociales es la docencia en espacios no universitarios, esta pregunta aborda elementos complejos, que se representan con la expresión de un estudiante de Licenciatura:

Muchos podemos saber historia, leyendo un libro se aprende historia, en un seminario usted aprende historia, pero ya cuando usted va a ingresar a un aula con distintas personas, con distintas mentalidades, con distintos contextos y épocas, saber enseñar historia es saber llegar a esa persona desde su contexto, desde la mentalidad de esa persona y eso de pronto uno sabiendo historia no tiene por qué conocerlo.

Este tipo de expresiones son reflejo y análisis de dos posturas importantes. Como primera medida, los estudiantes en su mismo proceso de formación reconocen que el neto saber disciplinar no es suficiente para explicar las situaciones de enseñanza y aprendizaje que se viven en las aulas de clase. Por otro lado, identificar el aprendizaje de la Historia como un proceso que se basa únicamente en la lectura, lo liga directamente a una historia factual y con poca necesidad de interpretación, por tanto existe una relación directa con las narrativas de carácter más positivista y tiende a excluir otras lógicas de pensamiento que no solo requieren de la formación mediante textos, sino en las posibilidades de debate que se desarrollan en narrativas de mayor crítica y multiplicidad de sentidos.

La situación mencionada por el estudiante de la Universidad de Caldas no se aísla de las condiciones expresadas por los Lineamientos Curriculares para las Ciencias Sociales, pues en este documento se identifica como problema y necesidad una situación en la que se "retoma en muchas ocasiones, la enseñanza de la historia, basada en fechas, y una geografía limitada a la descripción física de los lugares; es decir, donde los lugares son tratados como mapas exentos de contextos". Estas situaciones, para el caso específico de la Historia, tienden a contrastar con las perspectivas de muchos docentes universitarios, los que en sus discursos y prácticas exponen posturas de carácter crítico y comprometido con el contexto. La mencionada situación se expresa de manera más clara en las reflexiones de un docente de Licenciatura en Ciencias Sociales:
¿Cómo podemos cambiar la sociedad si no conocemos la historia? Ese es el rol de la historia, ¿cómo podemos nosotros generar procesos de movimientos sociales, cómo podemos generar posibilidades de cons- trucción de un país diferente, más justo, interesado por los desarrollos sociales, económicos y políticos si desconocemos la historia? Entonces, para cualquier área del conocimiento y no se trata de una for- mación superficial. Para nosotros generar situaciones de cambio, para mejorar la sociedad, la historia debe jugar un papel fundamental.

La postura del docente se relaciona directamente con ideas de historiadores como Fontana, para quien la Historia es un sistema de investigación que, mediante el desciframiento de su pasado, busca la comprensión de las razones que explican su situación presente y las perspectivas de que deben partir en la elaboración del futuro, de esta forma la Historia sería una herramienta en la capacidad de comprensión y de crítica.

Aunque las posturas de los docentes no son iguales, tienen ciertos puntos de convergencia, pues reconocen en la Historia no solo la posibilidad de aprender, sino reflexionar y ampliar opciones de pensamiento en el sujeto y la sociedad. Estas reflexiones pudieran relacionarse con postulados propios de la Escuela de los Annales, para quienes el objeto de la Historia trasciende del pasado mismo y se convierte en una posibilidad de relaciones con el presente desde el pasado, el pasado a partir del presente e incluso incluye el futuro como campo de acción de las reflexiones históricas, alrededor de marcos espaciales de menor amplitud pero mayor posibilidad de la construcción de sentido social e identidad, es decir, involucra asuntos regionales y locales.

Las premisas de comprensión de la Historia y sus lógicas científicas sociales se relacionan para casi todos los docentes y estudiantes con las posturas ya mencionadas de la Escuela de los Annales, pero en muchos de los casos el docente en formación no lo ejerce en sus procesos de acercamiento a la 
escuela y en su labor profesional. Surge entonces la cuestión: ¿por qué entonces existe esta brecha entre aquello que el estudiante aprende y lo que visualiza y aplica en sus prácticas?

Sin reconocer una única razón, el análisis de la información para el caso de la Universidad de Caldas permitió identificar, como posible situación explicativa, las múltiples divergencias y disputas entre la formación disciplinar y pedagógica, pues en aras de resaltar uno u otro componente, puede llegar a perderse la unidad requerida para que el docente en formación reconozca su labor. Como labor didáctica que es, requiere de ambos componentes, desarrollados de manera crítica y dialógica, sin el fin de llegar a recetas mágicas para la enseñanza de la Historia y las Ciencias Sociales, pero sí a una comprensión compleja sobre la misma, como un camino con múltiples rutas y formas de ser construido.

\section{La Didáctica de la Historia como puente de encuentro}

El estudio por la Didáctica ${ }^{14}$ de la Historia y las Ciencias Sociales cuenta con un trayecto interesante a partir del cual se han estructurado algunos grupos e instituciones, que entre algunas de sus líneas de investigación incluyen los procesos sobre la enseñanza y el aprendizaje de la temporalidad humana. La Didáctica se preocupa por las relaciones entre los procesos de enseñanza y aprendizaje frente a un campo del conocimiento, para el caso reúne las intencionalidades pedagógicas, políticas y formativas respecto de la necesidad de enseñar y aprender de las culturas de otros momentos y espacios, en aras de influir de uno u otro modo en el desarrollo de los sujetos y las comunidades del presente.

La Didáctica de la Historia se considera como un campo del conocimiento en construcción, cuya preocupación central son los procesos por los cuales el conocimiento histórico se convierte en un saber escolarizado, que a su vez responde a las características de docentes y educandos en contextos y realidades determinadas. Todo ello otorga al saber histórico un carácter de mayor amplitud al desarrollado netamente por los historiadores, pues el compromiso político, ético y social de la Historia como área escolar -o integrada en las Ciencias Sociales- es representativo y requiere por ello de un proceso de formación particular para el docente que va a enfrentarse a muchos de los problemas disciplinares, pero con mayor importancia a los problemas socialmente vivos que impactan a las comunidades.

Santisteban (2011) identifica el conocimiento de la historia como un pilar fundamental para la enseñanza de la historia, mas no el único factor a tener en cuenta. ${ }^{15}$ Esta reflexión se relaciona con posturas de docentes de Ciencias Sociales en ejercicio y formación dentro de la Universidad de Caldas:

\begin{abstract}
Uno debe tener conocimientos en Historia para poder enseñar, pero me parece muy fundamental saberlos transmitir. Es decir, no simplemente tener fechas y tener datos, personajes y acontecimientos, sino saber qué pasó después de estos acontecimientos y cómo estos cambiaron la vida del hombre en cualquier país o en cualquier momento de la Historia (Reflexión de un estudiante de Licenciatura en Ciencias Sociales).
\end{abstract}

En virtud de lo expuesto por el estudiante antes mencionado, bien vale la pena retomar a Carretero (1996: 104), quien indica que también es importante comentar que existen diferencias entre el conocimiento histórico y el propio de otras ciencias sociales. La historia supone un tipo de inteligencia diferente del conocimiento sociológico, económico o político. La tarea de la historia es coadyuvar a que el hombre desarrolle la comprensión de su devenir histórico y comprender el devenir histórico de la humanidad; es decir, que no se trata de delimitar fechas y narrar acontecimientos, se trata de comprender el porqué se dieron y de qué manera afectaron el desarrollo de los procesos sociales, económicos, políticos y culturales en determinada época y lugar; así como poder interpretar a su vez, cómo afectaron el presente y cómo pueden afectar el futuro de una región. Este aspecto también se evidencia en expresiones como la siguiente:

Usted tiene que conocer que tiene que tener condiciones diferentes si pretende enseñar por diferentes lugares sociales en Colombia, dado que usted no va enseñar Historia en el Caribe al igual que la va a enseñar en el Amazonas, entonces yo creo que saber enseñar historia es saber jugar o saber entender esas percepciones 
de los estudiantes o de las personas que están aprendiendo lo que usted les está enseñando (reflexión de un estudiante de Licenciatura en Ciencias Sociales).

Si bien resulta relevante conocer el contexto y el lugar donde se enseña, como un aspecto relevante en los procesos de enseñanza de la historia, también es muy cierto que cuando se enseña esta área, “... es crucial que el aprendizaje de la historia ayude a los alumnos a superar la óptica localista y presentista con que juzgan el mundo en que viven" (Domínguez 1989, p. 39); en tal perspectiva, es fundamental que los estudiantes no solo conozcan la historia y el contexto del lugar donde se encuentran, sino que también identifiquen "otras formas de vivir y trabajar, de relacionarse con la naturaleza y de organizarse en instituciones, otros modos de pensar, de sentir y de creer" (Díaz, F. 1988: 6). En otras palabras, es evidente que los contextos donde se enseña es fundamental, pero eso no puede obviar el que se tenga también conocimiento el contexto internacional, máxime en este mundo globalizado que estamos viviendo.

Estas reflexiones implican que "toda práctica de enseñanza de un objeto presupone, en efecto, la transformación previa de su objeto en objeto de enseñanza" (Verret en Gómez, 2005:84), y allí se incluye el concepto de transposición didáctica trabajado por Verret y Chevelard; para este último se remite "al paso del saber sabio al saber enseñado y luego a la obligatoria distancia que los separa. Hay de esta forma transposición didáctica cuando los elementos del saber pasan al saber enseñado" (Gómez, 2005: 87). La postura reconoce entonces como uno de los pilares de la Didáctica y por ende la Didáctica de la Historia el asunto disciplinar, pero a partir de un trascender del mismo en aras de lo educativo.

En cuanto a los fines didácticos de la Historia los docentes de la Universidad de Caldas han asumido el concepto de problematización, como lo menciona uno de ellos:

La historia debe enseñarse para resolver asuntos, el estudiante lo que necesita reconocer y analizar cómo ha sido ese proceso de la formación de una ciudad, no tanto desde los datos, desde las fechas, sino más bien desde los procesos de desarrollo de su entorno, porque la historia ayuda a problematizar, porque la historia ayuda a resolver problemas, la historia trae una conciencia, querámoslo o no del contexto social, político, económico en el que estamos inmergidos y estamos invitados a reflexionar y actuar (reflexiones de docente de Licenciatura en Ciencias Sociales).

Parece ser entonces que si la perspectiva anterior es una concepción generalizada entre los profesores del área de historia, es evidente que ellos, en sus prácticas docentes, reconocen la necesidad de problematizar la enseñanza y no dedicarse a la mera transmisión de información, aspecto que fundamentó durante mucho tiempo los procesos de enseñanza en las diversas áreas del conocimiento; en tal sentido, Acosta, L. (2003: 3), hablando de la historia, asume que el aprendizaje de la historia se ha fundamentado en un estereotipo social de carácter memorístico, no comprensivo, y que esta visión está arraigada y es aceptada por los propios discentes. Y continúa, apoyado en Fuentes (2004), que son excepcionales las experiencias de construcción de conocimiento orientadas a la interpretación original por los estudiantes, ni siquiera como ejercicio especulativo. Sin embargo, desde las concepciones de los docentes, de la Universidad de Caldas, es apreciable la preocupación por un proceso de enseñanza en el que el estudiante tenga un papel activo y crítico, en donde la transmisión no es la principal fuente que sustenta el proceso educativo en la Licenciatura en Ciencias Sociales.

\section{A manera de cierre}

Aprender para a enseñar Historia, en el caso de la Universidad de Caldas y quizás relacionado con las nuevas posturas de la Historia a nivel nacional y extranjero, es un asunto que requiere de formación en las diversas posturas del pensamiento histórico, principalmente en aquellas que se adhieren a un mayor nivel de compromiso con los contextos sociales y por tanto tiene mayor incidencia en el campo escolar. Estos asuntos, asumidos por muchos de los docentes formadores, son retomados por los educadores en su proceso de formación, sin embargo, cuentan con algunas distancias en los componentes formativos que dificultan las relaciones necesarias para convertir el saber sabio de la Historia en un saber enseñado y enseñable ${ }^{16}$ (Chevallard en Gómez, 2005) respecto de la misma, ya sea desde 
los discursos de la Historia Universal o Nacional o desde perspectivas de escritura y delimitación historiográfica diferentes a las tradicionales. ${ }^{17}$

Los supuestos acerca de la enseñanza y la Didáctica de la Historia que se han planteado hasta el momento en este texto, se han expresado de manera general, incluyendo tanto la historia de corte occidental como las posturas de historia latinoamericana y nacional; en estos contextos ha sido y es importante el estudio del porqué y desde qué perspectivas se estudia la enseñanza de los sucesos tempoespaciales abordados por la Historia y es quizás un debate sin consenso único, pero que sí puede asumir con uno de sus múltiples retos, el asunto de crear y construir alternativas de futuros a partir del conocimiento del pasado, situación que tiene sentido dentro de los contextos o espacios socialmente vivos en los cuales se desenvuelven los sujetos y se construye cultura.

Estas posibilidades de relacionar el tiempo y el espacio cambiantes en aras de comprender los contextos y transformarlos, presentan relaciones con la historia general, pero incluyen en sus dinámicas la construcción de una narrativa histórica desde lo micro, lo local y los significados en las comunidades no siempre incluidas en la narrativa histórica centralizada; dando espacio, también, al asunto de la Historia Regional, interés del presente trabajo. Ahora bien, La enseñanza de la región en términos históricos no puede ser estudiada por fuera de las posturas y retos que ha envuelto la enseñanza de la Historia en general, pero a su vez incluye ciertas particularidades, objetivos y posibilidades que podrán ser abordados en otros trabajos.

\section{Agradecimientos}

Para la Universidad de Caldas y Colciencias, instituciones que creyeron en esta idea y me ayudaron a consolidarla como propuesta de investigación, con su apoyo académico y financiero. Al Grupo de Investigación Maestros y Contextos por darle aval a las ideas de pensar en nuevas propuestas para la enseñanza de la Historia y las Ciencias Sociales. Para los maestros en ejercicio y formación que me hacen reflexionar cada día en para qué, cómo y por qué enseño Historia.

\section{Referencias Citadas}

Aisenberg, B. y Alderoqui S. (comps.) (

1994 Didáctica de las Ciencias Sociales. Aportes y Reflexiones. Buenos Aires. Paidós.

Arias, D

2014 La enseñanza de las ciencias sociales en Colombia: lugar de las disciplinas y disputa por la hegemonía de un saber. En: Revista de estudios sociales, número 52, Bogotá, Colombia.

Camilloni, A.

1994 Epistemología de la didáctica de las ciencias sociales. En Aisenberg, B. \& Alderoqui S. (1994). Didáctica de las Ciencias Sociales. Aportes y Reflexiones. Buenos Aires: Paidós.

Camillioni, A.

1995 "De lo cercano o inmediato" a "lo lejano" en el tiempo y el espacio. Revista del Instituto de Investigaciones en Ciencias de la Educación. Año IV ${ }^{\circ} 6$.

Carretero, $\mathrm{M}$.

1996 Construir y enseñar. Las ciencias sociales y la historia. Madrid: Aprendizaje Visor.

Carretero, M. y Kriger, M.

2010 Enseñanza de la historia e identidad nacional a través de las efemérides escolares. Buenos Aires, Paidós.

Díaz, F.

1998 Una aportación a la didáctica de la historia. La enseñanzaaprendizaje de habilidades cognitivas en el bachillerato. Revista Perfiles Educativos, $\mathrm{N}^{\circ} 82$ (págs. 40-66): Recuperado el 17 de mayo de 2014 de http://www.redalyc.org/articulo. oa? id=13208204.
Domínguez, J.

1989 El lugar de la Historia en el Currículum. Madrid: Aprendizaje Visor.

Gómez, M.

2005 La transposición didáctica: historia de un concepto. Revista Latinoamericana de Estudios Educativos. 1:83-115.

Guerrero, C.

2011 La incidencia de las reformas educativas en la enseñanza de la historia en Colombia: 1973- 2007. Tesis de Maestría. Maestría en Historia. Bogotá: Universidad Nacional.

Heyd, M.

2012 Is there a Future to the study of the Past?. Culture \& History Digital Journal 1(1): Recuperado el 13 de septiembre de 2014_en http://dx.doi.org/10.3989/chdj.

Jiménez, J. Sánchez, L.

2010 Enseñanza de la historia. Universidad de Caldas, Manizales. Documento sin publicar.

Lima, L., Bonilla, F. \& Arista, V.

2010 La enseñanza de la Historia en la escuela mexicana. Revista Proyecto Clío, (36): (1-16) . Recuperado el 17 de febrero de 2014 en http://clio.rediris.es/n36/articulos/ limaetalii.pdf.

Mangano, F. J.

2009 Contradicciones y discontinuidades: La Historia y su enseñanza. Revista de Teoría y Didáctica de las Ciencias Sociales, (15): 447-460.

Márquez, M.

2009 Enseñanza de la historia del conflicto armado en colombia: fundamentos para la construcción de propuestas 
para su enseñanza en el ambito universitario. Revista latinoamericana de Estudios Educativos, 5(2): 205-230). Mena, M.

2009 Dilemas sobre la enseñanza de la historia. Responsabilidad con la historia: Visibilizar e incluir la memoria de los grupos étnicos en el relato nacional. Revista Al tablero (51): (26-27).

Meyer, E.

2000 Transmisión de la conciencia histórica: memoria y conciencia histórica. Revista Historia, Antropología y Fuentes orales (24): 77-94.

Nosei, M. C.

2007 La enseñanza de la Historia en la escuela: una historia de malentendidos. Revista Praxis Educativa, 1: (74-83).

Pagés, J.

2004 Tiempos de cambios, ¿cambios de tiempos? Sugerencias para la enseñanza y el aprendizaje del tiempo histórico a inicios del siglo XXI. Retomado de: http://estudiantes.iems. edu.mx/cired/docs/ae/pp/hs/aepphspt01pdf02.pdf

Pagés, J., \& Santisteban, A.

2010 La enseñanza de la historia y el tiempo histórico en la educación primaria. Cad. Cedes, Campinas, 30 (82): 281-309.

Plá, S.

2005 Aprender a pensar históricamente, la escritura de la historia en el bachillerato. México D.F.: Plaza y Valdés Editores.

Posada, R. P.

2009 La enseñanza de la Historia: perspectivas y retos en la educación superior. Estudio de caso enseñanza de la historia en la licenciatura en ciencias sociales de la Universidad de Caldas. Tesis para optar al título de Magíster en Educación con énfasis en enseñanza de la Historia. Universidad de Caldas, Manizales. Colombia.

Prats, J.

2001 Enseñar Historia: notas para una didáctica renovadora. Mérida, México: Junta de Extremadura, Consejería de Educación, Ciencia y Tecnología.
Rivero, R.

2004 Memoria, Historia e Identidad. Una reflexión sobre la enseñanza de la historia en el desarrollo de la ciudadanía. En Carretero, M. \& Voss, J. (comps.), Aprender y Pensar la Historia. Buenos Aires: Amorrortu.

Rodríguez, S.

2012 Formación de maestros para el presente: memoria y enseñanza de la historia reciente. En: Revista colombiana de educación, número 62. Bogotá, Colombia.

Rüsen, J.

2001 What is Historical Consciousness? A Theoretical Approach to Empirical Evidence. Canadian Historical Consciousness in an International Context: Theoretical Frameworks, Vancouver, BC: University of British Columbia.

Rüssen. J.

2005 History: Narration, Interpretation, Orientation. Estados Unidos. Berghahn Books.

Rüssen, J.

2007 How to make sense of the past- salient issues of Metahistory. Journal of Transdisciplinary Research in Southern Africa, 3 (1).

Sandoval, C.

1996 La formulación y el diseño de los procesos de investigación social cualitativos: investigación cualitativa. Bogotá: ICFES.

Santisteban, A.

2010 La formación de competencias de pensamiento histórico. Revista Clío y Asociados (14): 34-56.

Santisteban, A.

2011 Conferencia Inagural y de apertura del II Encuentro Nacional de la Red Colombiana de Grupos de Investigación en Didáctica de las Ciencias Sociales. Septiembre 28 al 30, Manizales: Universidad de Caldas.

Valls, R.

1999 La didáctica de la historia en Alemania: una aproximación a sus características. Revista Didáctica de las Ciencias Sociales, Geografía e Historia (21): 89-105.

Zemelman, $\mathrm{H}$.

2007 El Ángel de la historia: determinación y autonomía de la condición humana. España: Editorial Anthropos.

\section{Notas}

1 Esta afirmación, que pudiera resultar prejuiciosa ante una óptica ligera, corresponde en realidad a los análisis realizados en el trabajo del estado del arte y del mismo modo a una lógica de pensamiento por parte de los profesionales en sus primeros años de ejercicio, ya que los discursos respecto de las transformaciones en la enseñanza resultan difíciles de articular con las prácticas reales de aula, además de las presiones institucionales y las pruebas que, a modo de ver de los egresados del programa, limitan de manera directa la labor de los docentes de Ciencias Sociales y de Historia.

2 El debate por la manera de ver y enseñar la Historia no corresponde únicamente a los docentes y licenciados, sino que es una reflexión asumida por los mismos historiadores, principalmente aquellos que se han involucrado en la Historia Social, como el caso de Hobsbawm (1983) al cuestionar acerca del rol social de la historia y de Marc Bloch (1982) al preguntarse, como eje de su libro Introducción a la Historia, ¿para qué sirve la historia? Desde el 2003 el profesor e historiador Carlos Barros ha liderado el Manifiesto por la defensa de la Historia, al que se han sumado gran cantidad de docentes y académicos del mundo, como parte de la Red Internacional Historia a Debate, en donde el asunto de la enseñanza y el compromiso social y ético juega un importante papel.

3 Es sustancial resaltar los contextos políticos como un eje central para pensar la enseñanza de la Historia, lo que se respalda desde las políticas de los Ministerios de Educación, quienes enmarcan la necesidad de vincular dichos contextos en los de formación de los educandos en el área de Ciencias Sociales. En el caso de Colombia desde los Lineamientos Curriculares, entendidos estos como orientaciones para que las instituciones -desde sus PEI- asuman la elaboración de sus propios currículos -para las Ciencias Sociales (MEN, 2002)-. Se propone que las Ciencias Sociales sean abordadas de manera integrada, sin embargo, en contextos como el argentino o el mexicano se trabaja la enseñanza de la Historia de manera diferenciada de los demás campos del saber social y humanístico.

4 Para Carretero, M. y Kriger, M. (2010: 57), cuando nos preguntamos por los "desafíos actuales" de la enseñanza de 
la historia, parece cada vez más difícil circunscribirnos a lo que sucede dentro de la escuela: las prácticas en el aula, los programas de estudio o los textos. Por el contrario, en los últimos quince años, lo que empieza como una eventual reforma de los libros de texto (como por ejemplo en México en 1992), o una reorientación de los lineamientos de los estándares de la asignatura (en EE. UU. en 1995), o una modificación (España en 1997), o, más recientemente, el intento de reescribir la historia nacional en un texto escolar único (en Rusia en 2007), puede derivar en un conflicto que rebalse ampliamente la práctica y el marco escolar. Esto nos muestra, por un lado, la centralidad creciente que está adquiriendo la historia escolar como arena de luchas identitarias y conflictos políticos en el mundo contemporáneo. Pero por otro lado también pone en evidencia hasta qué punto la propia enseñanza disciplinar, en tanto que práctica cognitiva y educativa, puede llegar a quedar por fuera de ese mismo escenario.

5 La Didáctica de la Historia alemana se ha movilizado alrededor de la categoría de conciencia histórica, la que es el eje fundamental de la propuesta de investigación y que se trabaja a profundidad en el tercer capítulo. Sin embargo es importante trabajarlo desde las ópticas de la enseñanza y la didáctica de la historia como un elemento importante dentro del estado del arte de la investigación. Según expone González, P. (2006: 24), originaria (aunque no exclusiva) del ámbito alemán, la categoría "Conciencia Histórica" comenzó a extenderse a los espacios académicos y ha sido central, especialmente a partir de los aportes teóricos de Jörn Rüsen, en la enseñanza de la historia.

6 Según Rüsen (1994: 3), esta nueva perspectiva categorial acerca de la historia en la vida de una sociedad no es una casualidad. Ha ido preparándose gracias a la cuestión de la conciencia histórica, que la didáctica de la historia ha tratado y discutido desde hace décadas para hablar del aprendizaje histórico -más allá de la enseñanza escolar de la historia- como un tema de significación general y fundamental y para reclamar esta cuestión como su campo de investigación propio. En el ámbito de la didáctica de la historia, la conciencia histórica ha recibido ya una significación categorial que sirve para la autoexplicación de la disciplina, para la identificación de una materia particular de estudio y de su metodología correspondiente y, al fin y al cabo, para la heurística de investigaciones empíricas.

7 Respecto de las construcciones teóricas propuestas por Rüssen (1994) se hablará con mayor detenimiento, pues el trabajo realizado por este autor se moviliza a partir de las categorías de Conciencia y Cultura Histórica, ejes principales de la propuesta de investigación y que se desarrollan en los capítulos tres y cuatro del presente trabajo. Ver: Rüsen, J. ¿Qué es la cultura histórica?: Reflexiones sobre una nueva manera de abordar la historia. Traducción de Sánchez, F. Disponible en: http://www.culturahistorica.es/ ruesen/cultura_historica.pdf. Recuperado julio 14 de 2014. Versión original en: : Füssmann, K., Grütter, H.T., Rüsen, J. (eds.): Historische Faszination. Geschichtskultur heute, 1994, pp. 3-26.

8 En palabras de González (2006: 21), la comprensión de la historia en los procesos de enseñanza en Argentina, "puede plantearse en términos de desarrollo de la percepción, interpretación y orientación históricas, piezas claves de la competencia narrativa. Aunque la conciencia histórica transmitida y construida en el aula es mucho más compleja que los contenidos de los textos, estos la documentan y permiten analizar las representaciones y competencias que se promueven. Bajo este supuesto de las representaciones sociales e identitarias es que se mueve la enseñanza de la historia en Argentina, según se evidencia en los textos escolares del nivel secundario editados con posterioridad a la reforma educativa de 1993.

9 Plá, S. (2012: 164), “[...] trata de enfrentar estas dos problemáticas de la siguiente manera: definir a la enseñanza de la historia como campo de investigación a partir de la relación que existe (íntima y extraña a la vez) entre la producción de conocimiento de la historiografía profesional y la historia enseñada mucho más allá de las fronteras universitarias. Esta interrelación es bastante más compleja de lo que se tiende a suponer, ya que puede ser un trampolín a la problematización de la enseñanza de la historia o ser un serio obstáculo para su desarrollo. Todo depende de cómo queramos concebir las fronteras disciplinares de la historia, si rígidas, estables y claramente definidas o flexibles, porosas e indefinidas Parte importante de la relación binaria investigación/enseñanza radica en las formas en que cada una de ellas produce el conocimiento histórico. Entre ellas hay semejanzas y diferencias que las unen pero que las hacen ser también particulares [...]".

10 La estructura que propone el MEN para el área de Ciencias Sociales, desde un enfoque interdisicplinar, expone que los Estándares Básicos de Competencias en Ciencias Sociales, se desarrolla a partir de tres tipos de relaciones: relaciones con la historia y las culturas, relaciones espaciales y ambientales y relaciones ético-políticas; relaciones que se deben asumir y desarrollar desde una mirada integral, a partir del enfoque interdisciplinario propuesto en los lineamientos del área, de manera que permitan una mejor comprensión de los fenómenos sociales, teniendo en cuenta los aportes conceptuales y metodológicos que ofrecen las variadas disciplinas que conforman las Ciencias Sociales. MEN. (2004: 121).

11 En la Revista SEMANA, se publicó un artículo el 24 de marzo de 2012, en el que se habla de la crisis de la Historia. Las consecuencias de haber desaparecido la cátedra de Historia en los colegios, unido a la baja calidad de los textos, han empezado a verse en la amnesia colectiva que se ha apoderado del país. Prestigiosos historiadores piden reversar esta absurda decisión. Colombia es uno de los países del mundo que menos atención y esfuerzo le pone a la enseñanza y al estudio de la Historia, tanto, que hoy la mayoría de los padres no saben que esta materia desapareció de los currículos. Por eso, casi 30 años después de que el Ministerio de Educación decidió sacar del pénsum de primaria y bachillerato la materia de Historia y crear la de Ciencias Sociales - una mezcla de Geografía, Economía, Política, Antropología, Sociología, Cultura e Historia-, un grupo de reconocidos historiadores e intelectuales le ha empezado a pedir al gobierno que, frente a la amnesia en la que cayó el país y ante semejante error pedagógico, permita de nuevo la enseñanza de la Historia como materia única de primero de primaria a grado once.

12 La Red de Grupos de Investigación en Didáctica de las Ciencias Sociales es una organización de carácter académico, 
con la pretensión de cualificar el campo de construcción científica referido a la enseñanza y el aprendizaje sobre lo social-humano, y tiene un recorrido de planeación y desarrollo de encuentros académicos e investigativos desde el 2010, como una idea mancomunada de varias instituciones de educación superior que ofertan programas relacionados con la formación de docentes de Ciencias Sociales o cuentan entre sus investigadores con personas reconocidas en los diversos tópicos de estudio. Entre sus principales líneas de trabajo se destacan: formación de educadores, conceptos de las Ciencias Sociales y sus problemas en el campo escolar, desarrollo del pensamiento social y formación ciudadana. Chevellard plantea la diferencia entre el saber "sabio" como parte de la construcción histórica de los saberes y el saber enseñado se relaciona con las determinaciones de diversa índole que parten al determinar qué de ese saber históricamente acumulado llega a la enseñanza obligatoria como parte de la formación de un colectivo de ciudadanos, que, se asume, requieren ese saber en su paso por la escuela. Uno de los principales debates que se generan actualmente en el campo de la Didáctica consiste en concebirla como un saber general o un saber específico en cada campo del conocimiento. La investigación se fundamenta en la postura de las didácticas específicas, para el caso la correspondiente a la Historia, pues las intencionalidades formativas se dan en relación con las mismas construcciones del mismo campo del saber. Sin embargo existen ciertos referentes generales necesarios de comprender en las Ciencias de la Educación, asumiendo la postura del Instituto Central de Ciencias Pedagógicas de Cuba (1991): "Es la teoría científica del proceso de enseñar y aprender, a partir de sus leyes y principios más generales, para ello tiene como categorías básicas los objetivos, el contenido, los métodos, los medios, las formas de organización y la evaluación, entre otras, que tiene en cuenta la unidad educación-formación-enseñanzaaprendizaje-desarrollo y la importancia de la comunicación en este proceso, en función de preparar al hombre para la vida, en un momento histórico social determinado".

15 Esta reflexión fue producto de la conferencia del profesor Antoni Santisteban en el marco de la apertura al II Encuentro Nacional de la Red Colombiana de Grupos de Investigación en Didáctica de las Ciencias Sociales, realizado en la Universidad de Caldas entre el 28 y el 30 de septiembre de 2011.

16 El concepto de transposición didáctica remite entonces al paso del saber sabio al saber enseñado y luego a la obligatoria distancia que los separa. Hay de esta forma transposición didáctica (en el sentido restringido) cuando los elementos del saber pasan al saber enseñado. Chevallard indica, en particular, que la transposición didáctica remite a la idea de una reconstrucción en las condiciones ecológicas del saber. Para ilustrar esta idea, él se vale de un ejemplo de transposición como el que sucede de una pieza musical del violín al piano: es la misma pieza, es la misma música, pero ella está escrita de manera diferente para poder ser interpretada con otro instrumento (Gómez, 2005:87).

17 Cuando se refiere a posturas no tradicionales de escritura de la Historia, se habla principalmente de aquellas pocas extendidas en el campo educativo como la Historia de las Mentalidades, la Historia de los de Abajo, la Microhistoria o la Historia Regional. 
